\title{
Diferenças no desempenho motor de crianças com excesso de peso e eutróficas
}

Differences in motor performance of overweight and eutrophic children

Manoella de Oliveira Santos

Programa de Pós-graduação em Ciência do Movimento Humano. Departamento de Aprendizagem Motora e

Desenvolvimento Motor. Universidade do Estado de Santa Catarina (UDESC), Brasil

Diego Grasel Barbosa

Observatório Educação Física e Saúde Coletiva. Universidade do Estado de Santa Catarina - Centro de Ciências da Saúde e do Esporte, Brasil

diegograsel1987@gmail.com

Andreia Pelegrini

Programa de Pós-graduação em Ciência do Movimento Humano. Departamento de Aprendizagem Motora e

Desenvolvimento Motor. Universidade do Estado de Santa Catarina (UDESC),Brasil

Raísa Carvalho da Silva

Programa de Pós-graduação em Ciência do Movimento Humano. Departamento de Aprendizagem Motora e

Desenvolvimento Motor. Universidade do Estado de Santa Catarina (UDESC),Brasil

Thaís Silva Beltrame

Programa de Pós-graduação em Ciência do Movimento Humano. Departamento de Aprendizagem Motora e

Desenvolvimento Motor. Universidade do Estado de Santa Catarina (UDESC),Brasil

\section{Érico Pereira Gomes Felden}

Programa de Pós-graduação em Ciência do Movimento Humano. Departamento de Aprendizagem Motora e

Desenvolvimento Motor. Universidade do Estado de Santa Catarina (UDESC),Brasil

\section{Resumo:}

Objetivou-se verificar as diferenças no desempenho nas tarefas do teste de proficiência motora de Bruininks-Oseretsky (TBO-2) entre crianças eutróficas e com excesso de peso. Participaram do estudo 98 crianças de oito a 10 anos regularmente matriculadas em três instituições particulares de ensino de Florianópolis-SC. Para avaliação do status de peso foram coletadas as variáveis de estatura e massa corporal e realizado cálculo do Índice de Massa Corporal (IMC). Para avaliação da proficiência motora foi utilizado TBO-2. Os dados foram analisados por meio de médias, desvios padrão, distribuição de frequência, histograma de distribuição dos dados, correlação de spearman e teste $\mathrm{U}$ de Mann Whitney para comparação dos valores médios entre os grupos. Verificaram-se correlações negativas do IMC com as tarefas de transferir moedas ( $\mathrm{r}=-0,311$, $\mathrm{p}=0,002)$, salto sincronizado $(\mathrm{r}=-0,201 ; \mathrm{p}=0,047)$, flexão de braço $(\mathrm{r}=-0,240 ; \mathrm{p}=0,017)$ e pontuação total do TBO-2 ( $\mathrm{r}=-0,243 ; \mathrm{p}=0,016)$. Além disso, observaram-se melhores desempenhos nas tarefas de transferir moedas $(p=0,026)$, salto sincronizado $(p=0,030)$ e flexão de braço $(p=0,019)$ nas crianças eutróficas comparadas às crianças com excesso de peso. Embora verificaram-se que crianças com excesso de peso apresentaram pior desempenho no escore geral de proficiência motora, esta diferença foi maior em tarefas motoras específicas que envolvem as capacidades motoras de força de membros superiores, coordenação e equilíbrio.

Palavras-Chave: Crianças, Estado nutricional, Habilidades motoras, Performance motora.

\begin{abstract}
:
The aim of this study was to verify performance differences in the tasks of Bruininks-Oseretsky motor proficiency test (TBO-2) among eutrophic and overweight children. A total of 98 children aged 8 to 10 enrolled in three private educational institutions in Florianópolis-SC participated in the study. To evaluate the weight status, the variables of height and body mass were collected and the Body Mass Index (BMI) was calculated. TBO-2 was used to assess motor proficiency. Data were analyzed using means, standard deviations, frequency distribution, histogram of data distribution, spearman correlation and Mann Whitney $U$ test for comparison of mean values between groups. There were negative correlations of BMI with the tasks of transferring coins $(r=-0.311, p=0.002)$, synchronous jump $(r=-0.201, p=0.047)$, arm flexion $(r=$ $-0.240, p=0.017)$ and total TBO-2 score $(r=-0.243 ; p=0.016)$. In addition, there were better performances in the tasks of transferring coins $(p=0.026)$, synchronous jump ( $\mathrm{p}=0.030)$ and arm flexion $(\mathrm{p}=0.019)$ in eutrophic children compared to overweight children. Although overweight children were found to perform poorly on the overall motor proficiency score, this difference was greater in specific motor tasks involving motor skills of upper limb strength, coordination and balance.
\end{abstract}

KEYWORDS: Child, Nutritional status, Motor skills, Motor performance.

\section{INTRODUÇÃo}

De acordo com a Organização Mundial de Saúde, a obesidade é uma epidemia de escala global e considerada um grave problema de saúde pública (WHO, 2004). As prevalências de obesidade têm aumentado nas últimas décadas em todas as faixas etárias e, em especial, na infância. Até 2011 era estimado que pelos menos $25 \%$ das pessoas com idade inferior a 18 anos apresentavam excesso de peso (Swinburn et al., 2011). Além disso, a atenção para a obesidade na infância vem ganhando destaque considerando que crianças e adolescentes obesos apresentam cinco vezes mais propensão a obesidade na vida adulta, de modo

Recepción: 28 Mayo 2020 | Aprobación: 15 Septiembre 2020 | Publicación: 30 Septiembre 2020 
que $55 \%$ das crianças obesas passam a ser adolescentes obesos e, aproximadamente $80 \%$ dos adolescentes obesos se tornam adultos obesos (Simmonds, Llewellyn, Owen \& Woolacott, 2016).

Estudos têm apresentado associações da obesidade com diversas doenças como hipertensão arterial (Rosaneli et al., 2014), problemas cardiovasculares (Gazolla et al., 2014), diabetes mellitus tipo II (Hannon, Rao \& Arslanian, 2005) e problemas de coluna (Kussuki, João \& da Cunha, 2007). Diante disso, é de se esperar que a obesidade também traga repercussões negativas para o desenvolvimento motor considerando diferentes lógicas de análise. Se, por um lado, o aumento da gordura corporal possa ser associado com dificuldades de controle dos movimentos, deslocamentos e dificuldade no uso adequado de força, por outro lado, considera-se que a obesidade se constitua numa restrição individual funcional na medida em que crianças obesas possam sofrer com estereótipos se inserindo menos em atividades físicas e esportivas por vergonha de sua forma corporal (Wade \& Whiting, 1986; Pereira, Graup, Lopes, Borgatto \& Daronco, 2009).

Considerando possíveis diferenças no desempenho motor de crianças com peso saudável e obesas, grande parte dos estudos da literatura identificaram associação entre obesidade e baixos índices de desempenho motor com a utilização de diferentes instrumentos de medida, como o Movement Assessment Battery for Children (MABC-2) (D’Hondt, Deforche, De Bourdeaudhuij \& Lenoir, 2009; Miranda, Beltrame \& Cardoso, 2011; Logan, Scrabis-Fletcher, Modlesky \& Getchell, 2011) o Körperkoordination Test fürKinder (KTK) (D’Hondt et al., 2011; D’Hondt et al., 2013; D’Hondt et al., 2014; Lopes, Stodden, Bianchi, Maia, \& Rodrigues, 2012) o Test of Gross Motor Development-2 (TGMD-2) (Cliff et al., 2012; Zequinão et al., 2015; Zanella, Bandeira, de Souza \& Valentini, 2015) e o Bruininks-Oseretsky Test of Motor Proficiency, second edition (TBO-2) (Gentier et al., 2013; Nunez-Gaunaurd, Moore, Roach, Miller \& Kirk-Sanchez 2013; Marmeleira, Veiga, Cansado \& Raimundo, 2017).

Em estudos que utilizaram o instrumento MABC-2 para avaliar o desempenho e habilidades motoras, os autores analisaram as diferenças no desempenho motor entre grupos de crianças com peso saudável e excesso de peso a partir dos domínios (destreza manual, habilidades com bola e equilíbrio dinâmico e estático) (D'Hondt et al., 2009; Miranda, Beltrame \& Cardoso, 2011) e a partir dos percentis do escore total do teste de acordo com o sexo e idade (Logan et al., 2011) Nesta mesma linha de raciocínio, estudos que utilizaram o KTK, para investigar associações entre estado nutricional e desempenho motor de crianças, utilizaram o índice de quociente motor (proficiência motora grossa), em detrimento da comparação das tarefas especificas do instrumento (D’Hondt et al., 2011; Lopes et al., 2012; D’Hondt et al., 2013; D’Hondt et al., 2014). Acredita-se, no entanto, que análises comparativas que considerem as pontuações de cada tarefa da proficiência motora possam ser mais precisas e direcionadas para intervenções e propostas de atividades para grupos de indivíduos identificados com dificuldades motoras específicas.

Neste sentido, Gentier et al. (2013), buscaram analisar as diferenças tanto dos domínios quanto das tarefas motoras finas e grossas entre crianças obesas e com peso saudável por meio do TBO-2. Os autores verificaram que crianças obesas apresentaram pior desempenho em todas as tarefas motoras grossas e em algumas tarefas motoras finas (destreza manual e precisão motora) comparadas aos seus pares de peso saudável. Apesar de ser bem documentado na literatura que crianças com excesso de peso e/ou obesidade apresentam piores desempenhos em tarefas que envolvem habilidades motoras grossas (D'Hondt et al., 2013; D'Hondt et al., 2011; Okely \& Booth, 2004; Okely, Booth, \& Chey, 2004; Poulsen et al., 2011), alguns estudos têm verificado que além da proficiência grossa, crianças com maior IMC apresentam também piores desempenhos em tarefas envolvendo proficiência motora fina (Petrolini, Iughetti \& Bernasconi, 1995; D'Hondt et al., 2009; Gentier et al., 2013).

Desta forma, aponta-se a importância em avaliar e aprofundar a investigação da relação do excesso de peso com cada tarefa motora, tanto de habilidades motoras grossas quanto finas em amostras de crianças de oito a 10 anos, tendo em vista que, a maioria dos estudos buscaram analisar o desempenho motor agrupando as tarefas em domínios ou avaliando apenas habilidades motoras grossas. Sendo assim, o presente estudo teve 
como objetivo verificar as possíveis diferenças no desempenho em cada uma das tarefas do teste TBO-2 entre crianças eutróficas e com excesso de peso.

\section{MÉTODo}

\section{Participantes do estudo}

O estudo foi realizado com uma amostra intencional de 98 crianças de oito a 10 anos, provenientes de três escolas privadas de Florianópolis-SC. Trata-se de um estudo de corte transversal com amostragem do tipo não probabilística intencional (Gaya, 2008). Foram enviados aos pais/responsáveis cartilhas de apresentação contendo informações sobre os procedimentos e objetivos da pesquisa, juntamente com o Termo de Consentimento Livre e Esclarecido (TCLE). Participaram do estudo as crianças cujos pais/ responsáveis autorizaram por meio da assinatura do termo. O estudo foi aprovado pelo Comitê de Ética em Pesquisa com Seres Humanos da Universidade do Estado de Santa Catarina (Protocolo 1.520.804/2016).

\section{Variáveis Investigadas}

A proficiência motora foi avaliada por meio do teste de proficiência motora de Bruininks-Oseretsky, 2a Edição (TBO-2) (Bruininks \& Bruininks, 2005). Foi utilizada a versão curta do teste composta por 14 tarefas divididas em 8 subtestes: precisão motora fina (desenhar o caminho e dobrar o papel), integração motora fina (copiar o quadrado e a estrela), destreza manual (transferir moedas), coordenação bilateral (salto sincronizado no lugar e toque sincronizado), equilibro (andar sobre a linha e ficar em pé na trave) corrida, velocidade, agilidade (salto unipodal), coordenação de membros superiores (largar e pegar a bola e driblar a bola), e força (flexão de braço e abdominal).

As pontuações das tarefas de precisão motora fina variam de 0 a 7 pontos; integração motora fina de 0 a 5 pontos; destreza manual de 0 a 9 pontos; equilíbrio de 0 a 4 pontos; corrida, velocidade, agilidade de 0 a 10 pontos; força de 0 a 9; quanto a coordenação bilateral, o salto sincronizado no lugar varia de 0 a 3 pontos e o toque sincronizado de 0 a 4 pontos; para coordenação de membros superiores, largar e pegar a bola varia de 0 a 5 e driblar a bola varia de 0 a 7 . De acordo com a realização destas tarefas atribuiu-se uma pontuação. $\mathrm{O}$ somatório de todas as tarefas possibilita uma pontuação que varia de 0 a 88 pontos. A partir dessa pontuação levando em consideração a idade de cada indivíduo, obteve-se o escore total da proficiência que variou de 20 a 80 pontos.

Para aferição do Índice de Massa Corporal (IMC) foram coletadas medidas de massa corporal e estatura, de acordo com os procedimentos de Alvarez e Pavan (2003). As medidas de massa corporal e estatura forma coletadas em ambiente reservado. Para aferir a massa corporal utilizou-se balança da marca Tanita com capacidade de até $150 \mathrm{~kg}$. A estatura foi aferida com uma fita métrica fixada na parede com resolução de $0,1 \mathrm{~cm}$. O status de peso foi classificado de acordo com os pontos de corte recomendados por Cole, Flegal, Nicholls y Jackson (2007). As crianças foram classificadas em dois grupos em relação ao status de peso: eutróficos, grupo que incluiu indivíduos com peso normal de acordo com a idade e seis indivíduos que apresentaram baixo peso. E o grupo com excesso de peso, no qual compreendeu indivíduos com sobrepeso e obesidade.

Tratamento de dados

A análise dos dados foi feita a partir do programa estatístico Statistical Package for Social Sciences (SPSS) versão 20.0. adotando-se um nível de significância de 5\%. Os dados foram analisados por meio de medidas descritivas em médias, desvios padrão, distribuição de frequência e histograma de distribuição dos dados. Foram utilizadas também medidas inferenciais como, correlação de spearman e comparação dos valores médios entre os grupos por meio do teste $\mathrm{U}$ de Mann-Whitney. Quanto à análise do ranking de tarefas 
foi realizada a padronização das pontuações das tarefas motoras por meio de escore z. Adotou-se para classificação da proficiência motora os seguintes valores: 2 para muito acima da média, 1 para acima da média, 0 para indivíduos na média, -1 para indivíduos abaixo da média e -2 para muito abaixo da média. Em seguida, foi verificado a frequência de indivíduos com pontuação inferior ou igual a -1 em cada tarefa, tanto no grupo de eutróficos quanto no grupo de excesso de peso. Por fim, foram selecionadas cinco tarefas com maior frequência de indivíduos que apresentaram pontuação inferior à média em ambos os grupos.

\section{Resultados}

A amostra foi composta por $61,2 \%$ de meninas, com média de idade de $9,49(0,8)$ anos. A média de IMC do grupo foi $18,88 \mathrm{~kg} / \mathrm{m}^{2}$, sendo $40,8 \%$ da amostra com excesso de peso (Tabela 1). Ainda na Tabela 1 , foram apresentadas as médias de cada tarefa motora do grupo geral.

TABELA 1

Dados descritivos da amostra.

\begin{tabular}{|l|c|}
\hline Variáveis & Geral $^{*}$ \\
\hline Sexo feminino, $\mathrm{n}(\%)$ & $60(61,2)$ \\
\hline Idade, anos & $9,49(0,8)$ \\
\hline IMC, $\mathrm{kg} / \mathrm{m}^{2}$ & $18,88(3,2)$ \\
\hline Status de peso, $\mathrm{n}(\%)$ & \\
$\quad$ Eutrófico & $58(59,2)$ \\
$\quad$ Excesso de peso & $40(40,8)$ \\
\hline Tarefas TBO-2, pontos & \\
\hline Desenhar o caminho & $6,87(0,3)$ \\
Dobrar o papel & $6,31(1,3)$ \\
\hline Copiar quadrado & $4,91(0,3)$ \\
Copiar estrela & $4,33(1,4)$ \\
\hline Transferir moedas & $4,50(1,4)$ \\
\hline Salto sincronizado & $2,58(0,6)$ \\
Toque sincronizado & $3,89(0,3)$ \\
\hline Andar sobre a linha & $3,99(0,1)$ \\
\hline Ficar em pé na trave & $3,55(0,9)$ \\
\hline Salto unipodal no lugar & $8,08(0,5)$ \\
\hline Largar e pegar a bola & $4,76(0,5)$ \\
Driblar a bola & $5,22(1,9)$ \\
\hline Flexão de braço & $3,56(1,3)$ \\
Abdominal & $4,11(0,9)$ \\
\hline TBO-2, escore & $45,53(6,3)$ \\
\hline
\end{tabular}

* Valores expressos em média desvio padrão para variáveis continuas e $\mathrm{n}^{\circ}$ percentual para variáveis categóricas TBO-2 teste de proficiência motora de Bruininks-Oseretsky

Observaram-se correlações significativas entre o IMC e as tarefas de transferir moedas $(r=-0,311$; $\mathrm{p}=0,002)$, salto sincronizado $(\mathrm{r}=-0,201 ; \mathrm{p}=0,047)$, flexão de braço $(\mathrm{r}=-0,240 ; \mathrm{p}=0,017)$ e escore total do TBO-2 ( $\mathrm{r}=-0,243 ; \mathrm{p}=0,016)$ (Tabela 2). 
TABELA 2

Correlação entre IMC e as tarefas do teste de proficiência motora.

\begin{tabular}{|l|c|c|}
\hline \multirow{2}{*}{ Tarefas TBO-2 } & rho & IMC-valor* \\
\cline { 2 - 3 } & $-0,115$ & 0,258 \\
\hline Desenhar o caminho & $-0,041$ & 0,687 \\
Dobrar o papel & $-0,012$ & 0,908 \\
\hline Copiar quadrado & 0,017 & 0,871 \\
Copiar estrela & $-0,311$ & 0,002 \\
\hline Transferir moedas & $-0,201$ & 0,047 \\
\hline Salto sincronizado & $-0,043$ & 0,657 \\
Toque sincronizado & 0,088 & 0,389 \\
Andar sobre a linha & $-0,177$ & 0,081 \\
Ficar em pé na trave & $-0,048$ & 0,637 \\
\hline Salto unipodal no lugar & $-0,036$ & 0,722 \\
\hline Largar e pegar a bola & $-0,113$ & 0,266 \\
Driblar a bola & $-0,240$ & 0,017 \\
\hline Flexão de braço & $-0,066$ & 0,519 \\
Abdominal & $-0,243$ & 0,016 \\
\hline TBO, escore & & \\
\hline
\end{tabular}

* p-valor do teste de correlação de Sperman

A partir dos resultados encontrados na análise de correlação, foram realizadas análises de comparação entre médias, comparando as médias nas tarefas de proficiência motora entre crianças eutróficas e com excesso de peso. Na Figura 1 foram apresentadas as tarefas que apresentaram diferença significativa entre indivíduos com excesso de peso e eutróficos. Verificaram-se maiores médias nas tarefas de transferir moedas $(\mathrm{p}=0,026)$, salto sincronizado $(\mathrm{p}=0,030)$ e flexão de braço $(\mathrm{p}=0,019)$ nas crianças eutróficas quando comparadas aos seus pares com excesso de peso (Figura 1).

\section{FIGURA 1}

Diferença entre os valores médios das tarefas do teste de proficiência motora em relação ao status de peso. Teste U de Mann Whitney.

Eutrófico Excesso de peso

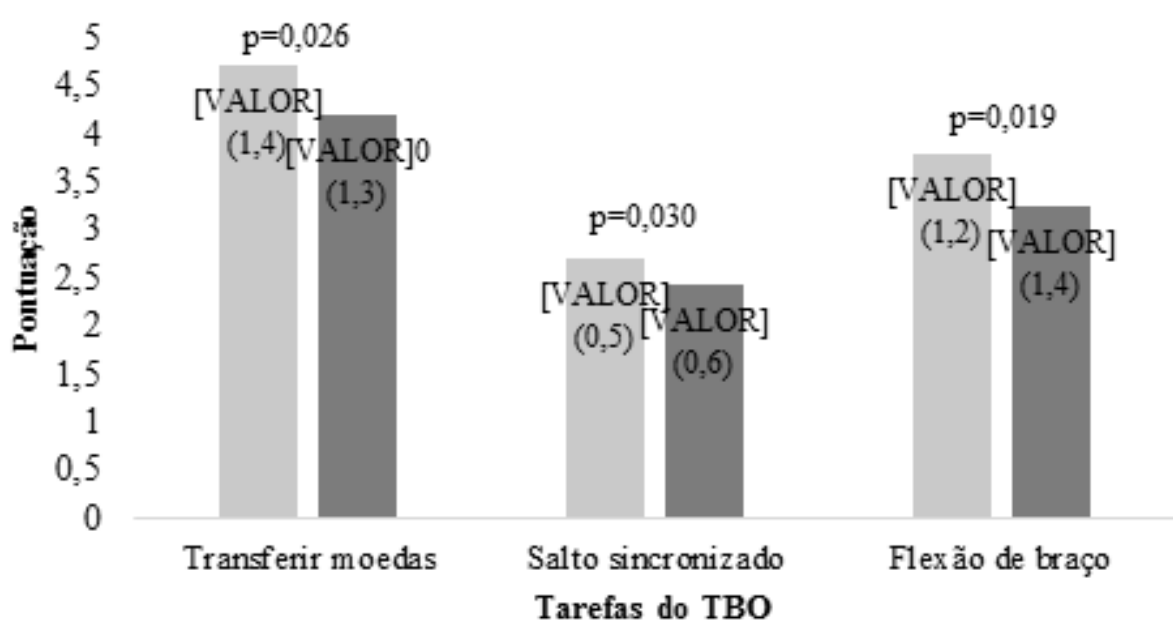

Na Figura 2 foram apresentados histogramas das tarefas motoras nas quais foram observadas correlações e diferenças entre médias nos grupos de eutróficos e com excesso de peso. No eixo Y apresentam-se a 
frequência de indivíduos, já no eixo $\mathrm{X}$ a pontuação obtida na tarefa. Observaram-se que para as tarefas de transferir moedas, salto sincronizado e flexão de braço os indivíduos eutróficos apresentaram assimetria negativa $(-0,832 ;-1,264 ;-0,603$ respectivamente). Assim, poucos indivíduos eutróficos apresentaram escores mais baixos nestas tarefas, sendo a concentração de indivíduos maior no canto direito do gráfico. Em relação ao grupo com excesso de peso, observou-se uma concentração maior nas pontuações medianas em todas as tarefas, sendo os valores de assimetria de $-0,237$ para tarefa de transferir moedas, $-0,649$ para o salto sincronizado e -0,090 para flexão de braço.

FIGURA 2

Distribuição da frequência de indivíduos em cada pontuação obtida nas tarefas de transferir moedas, salto sincronizado e flexão de braço de crianças eutróficas e com excesso de peso.
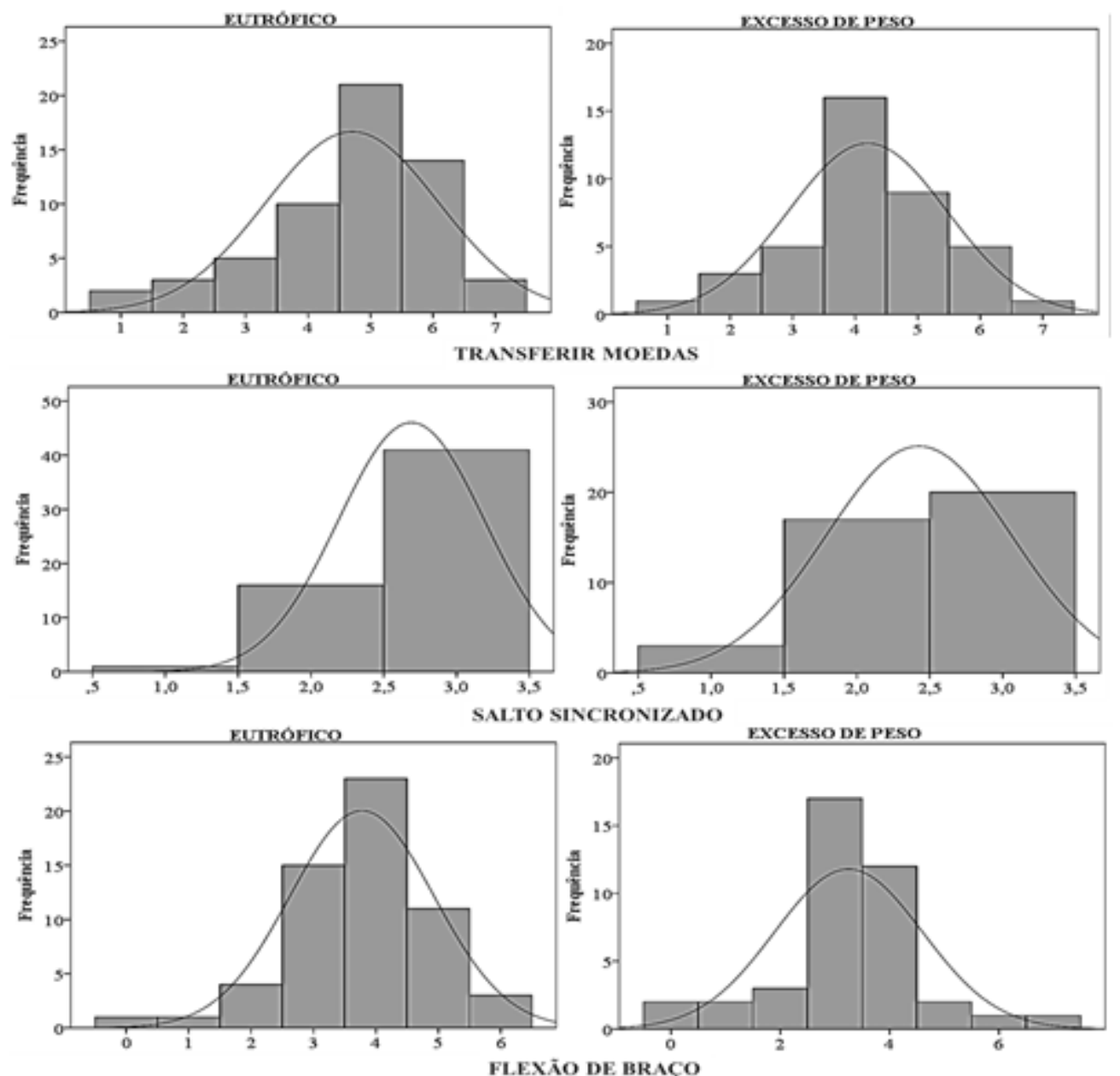

Ao analisar quais tarefas de proficiência motora os indivíduos apresentam mais dificuldades, verificouse que ambos os grupos apresentaram mais dificuldade nas tarefas de salto sincronizado, transferir moedas, dobrar o papel, driblar a bola alternando as mãos e largar e pegar a bola. No entanto, o grupo com excesso de peso apresentou dificuldade em mais algumas tarefas além dessas, são elas: equilíbrio na barra, flexão de braço e desenhar o caminho (Quadro 1). 


\section{QUADRO 1}

Ranking das tarefas de proficiência motora em que os indivíduos obtiveram pontuações inferiores à média.

\begin{tabular}{|c|c|c|}
\hline \multirow{2}{*}{ Colocação } & Eutrófico & Excesso de peso \\
\cline { 2 - 3 } & \multicolumn{2}{|c|}{ Tarefas } \\
\hline $1^{\circ}$ & Salto sincronizado & Salto sincronizado \\
\hline $2^{\circ}$ & Driblar a bola alternando as mãos & Driblar a bola alternando as mãos \\
\hline $3^{\circ}$ & Dobrar o papel & $\begin{array}{c}\text { Equilíbrio na barra } \\
\text { Largar e pegar a bola } \\
\text { Transferir moedas }\end{array}$ \\
\hline $4^{\circ}$ & Largar e pegar a bola & Desenhar caminho \\
\hline $5^{\circ}$ & Transferir moedas & $\begin{array}{c}\text { Dobrar o papel } \\
\text { Flexão de braço }\end{array}$ \\
\hline
\end{tabular}

\section{Discussão}

O presente estudo teve como objetivo verificar as possíveis diferenças no desempenho em cada uma das tarefas do TBO-2 entre crianças eutróficas e com excesso de peso. Verificou-se que o IMC apresentou correlação negativa com três tarefas individuais do teste de proficiência motora (transferir moedas, salto sincronizado e flexão de braço) e com a pontuação total do TBO-2. Observaram-se também, maiores médias de pontuações nessas mesmas tarefas nas crianças eutróficas quando comparadas às crianças com excesso de peso. Além disso, foi verificado que, tanto as crianças eutróficas como as crianças com excesso de peso apresentaram dificuldades em determinadas tarefas. Porém identificaram-se tarefas que apenas as crianças com excesso de peso tiverem dificuldade, como equilíbrio na barra, desenhar um caminho e flexão de braço.

No presente estudo observou-se que crianças com excesso de peso apresentaram menores médias de pontuação na tarefa de salto sincronizado com relação às crianças eutróficas. Este achado vai ao encontro dos estudos de Gentier et al. (2013) e Collet, Folle, Pelozin, Botti e do Nascimento (2008), nos quais verificaram piores pontuações de crianças obesas nas tarefas de salto sincronizado e na coordenação motora respectivamente comparadas às crianças com peso saudável. A tarefa de salto sincronizado exige, além da coordenação corporal, o equilíbrio dinâmico para manter a posição durante e entre os saltos. Neste sentido, estudos apontam que crianças eutróficas desempenham melhor as tarefas de equilíbrio do que crianças obesas (Berleze, Haeffner \& Valentini, 2007; Gentier et al., 2013), corroborando com o presente estudo, no qual crianças com excesso de peso também apresentaram mais dificuldades para realização da tarefa de equilíbrio estático na barra em relação aos seus homólogos eutróficos.

Desta forma, levando em consideração que ambas tarefas (salto sincronizado e equilíbrio estático na barra) envolvem a necessidade do equilíbrio, acredita-se que a explicação para ambos resultados encontrados seja devida as alterações posturais decorrentes do excesso de peso (Kussuki, João \& Cunha, 2007) que interferem na localização do centro de gravidade do corpo (Sibella, Galli, Romei, Montesano \& Crivellini 2003). Estes fatores por sua vez, dificultam a percepção do ponto de equilíbrio e, deste modo, intervêm na realização de tarefas que exijam esta capacidade motora.

Além das tarefas de equilíbrio e coordenação corporal, verificou-se que crianças com excesso de peso apresentaram médias inferiores na tarefa de flexão de braço em relação às crianças eutróficas $(p=0,019)$. A tarefa de flexão envolve a necessidade de controle corporal e principalmente a força de membros superiores. Berleze, Haeffner e Valentini (2007), observaram resultados semelhantes, no qual crianças obesas possuíam menos força de membros superiores que as eutróficas, pois não conseguiam realizar um arremesso tão longe quanto seus pares de peso saudável. Outro estudo (Gentier et al., 2013) que avaliou força de membros superiores por meio da mesma tarefa de flexão de braço, encontrou o mesmo resultado do presente estudo. 
Supõe-se que indivíduos com excesso de peso apresentem mais dificuldade na tarefa de flexão de braço, pois possuem maior quantidade de massa corporal repercutindo assim em uma necessidade de maior força de membros superiores para sustentar o corpo em relação aos indivíduos eutróficos.

No presente estudo, além das tarefas que apresentaram relação com o excesso de peso citadas anteriormente, observaram-se também relações do excesso de peso com tarefas de transferir moedas (destreza manual) e desenhar o caminho (precisão motora fina). Em estudo realizado por Gentier et al. (2013), foi observado semelhante, no qual crianças obesas também apresentaram menores escores para a tarefa de destreza manual e para o conjunto de tarefas de precisão motora fina. Outros estudos, como os de D'Hondt et al. (2009) também verificaram a relação negativa entre obesidade e habilidades motoras finas. Levandose em consideração que a quantidade de massa corporal de indivíduos com excesso de peso não deveria interferir na realização das habilidades motora finas, supõe-se que a relação entre estas variáveis ocorra devido a outras questões de natureza neurológica que possam envolver o controle motor. Petrolini, Iughetti \& Bernasconi et al. (1995) sugerem que crianças obesas experimentem dificuldades com a integração e processamento de informações sensoriais, quando as mesmas são necessárias para planejar e controlar o movimento. Contudo, essas questões não estão claramente apresentadas na literatura necessitando, desta forma, maiores aprofundamentos.

Além disso, possivelmente crianças com excesso de peso atinjam escores inferiores de proficiência motora em relação a crianças eutróficas pelo fato não realizarem determinadas tarefas no dia-a-dia por vergonha de se exporem ou por não se sentirem capazes de realizar a tarefa da mesma maneira que seus pares. Esta hipótese se confirma no estudo de Carissimi et al. (2017), no qual crianças com um IMC mais elevado, apresentaram menores pontuações da escala de auto eficácia. Neste sentido, uma baixa auto eficácia pode resultar em um não encorajamento de crianças com excesso de peso na realização de atividades em que iriam expô-las em público, fazendo com que as mesmas não consigam atingir proficiência motora adequada em determinadas tarefas devido à falta de prática das mesmas.

Acredita-se com isso, que o encorajamento de pais e professores para o desenvolvimento de fatores psicológicos como autoconfiança e auto eficácia nas habilidades e capacidades motoras de crianças com excesso de peso, poderia diminuir as dificuldades em tarefas motoras específicas, além de estimular maior prática e envolvimento em atividades físicas e esportivas por estes sujeitos. Neste sentido, D'hondt et al. (2014), verificaram em estudo longitudinal composto por 2517 crianças de 5 a 13 anos que um menor desempenho motor na baseline previu significativamente um aumento no IMC $(B=-0,003, p=0,027)$ e o IMC mais alto na baseline também previu uma diminuição no desempenho motor ao final dos dois anos de estudo $(\mathrm{B}=-1,792, \mathrm{p}<0,001)$.

O presente estudo contribui para a área de Educação Física e Esporte, no sentido de apontar e discutir sobre dificuldades tarefas motoras grossas e finas específicas de crianças com excesso de peso comparadas às crianças com peso normal. Neste sentido, acredita-se que o desenvolvimento de habilidades motoras fundamentais atrelado ao fortalecimento de variáveis psicológicas como auto eficácia, autoconfiança e auto estima devem ser uma das prioridades nas aulas de Educação Física escolar dos anos iniciais do Ensino Fundamental.

Contudo, apontam-se como limitações o corte transversal e o tamanho da amostra relativamente pequeno, o que dificulta a generalização desses achados para a população de crianças de oito a 10 anos da cidade investigada. Desta forma, sugere-se a realização de estudos com amostras epidemiológicas de crianças com e sem excesso de peso, que tenham como objetivo investigar de que forma a dificuldade em tarefas motoras pode interferir no dia-a-dia destas crianças. Além disso, propõe-se a realização de estudos com enfoque neurológico para compreender o porquê crianças com excesso de peso apresentam mais dificuldades em algumas habilidades motoras finas em relação às crianças eutróficas. 


\section{Conclusão}

Diante dos resultados observou-se que crianças com excesso de peso apresentaram médias significativamente inferiores nas tarefas de transferir moedas, salto sincronizado e flexão de braço em relação às crianças eutróficas. Além disso, observou-se que as crianças com excesso de peso apresentaram mais dificuldades na realização das tarefas de equilíbrio na barra, desenhar um caminho e flexão de braço do que seus pares eutróficos. Conclui-se que é necessária atenção especial para o monitoramento e acompanhamento do desenvolvimento e desempenho motor de crianças com e sem excesso de peso inseridas nos ambientes de Educação Física escolar.

\section{REFERÊNCIAS}

Alvarez, B. R., \& Pavan, A. L. (2003). Alturas e comprimentos. Antropometria: técnicas e padronizações, 2, 59-71.

Berleze, A., Haeffner, L. S. B., \& Valentini, N. C. (2007). Desempenho motor de crianças obesas: uma investigação do processo e produto de habilidades motoras fundamentais. Rev Bras Cineantropom Desempenho Hum, 9(2), $134-44$.

Bruininks, R. H. (2005). Bruininks-Oseretsky test of motor proficiency. AGS Publishing Circle Pines, MN.

Carissimi, A., Adan, A., Tonetti, L., Fabbri, M., Hidalgo, M. P., Levandovski, R., ... \& Martoni, M. (2017). Physical self-efficacy is associated to body mass index in schoolchildren. Jornal de pediatria, 93(1), 64-69.

Cliff, D. P., Okely, A. D., Morgan, P. J., Jones, R. A., Steele, J. R., \& Baur, L. A. (2012). Proficiency deficiency: mastery of fundamental movement skills and skill components in overweight and obese children. Obesity, 20(5), 1024-1033.

Cole, T. J., Flegal, K. M., Nicholls, D., \& Jackson, A. A. (2007). Body mass index cut offs to define thinness in children and adolescents: international survey. Bmj, 335(7612), 194.

Collet, C., Folle, A., Pelozin, F., Botti, M., \& do Nascimento, J. V. (2008). Nível de coordenação motora de escolares da rede estadual da cidade de Florianópolis. Motriz. Journal of Physical Education. UNESP, 373-380.

D’Hondt, E., Deforche, B., De Bourdeaudhuij, I., \& Lenoir, M. (2009). Relationship between motor skill and body mass index in 5-to 10-year-old children. Adapted Physical Activity Quarterly, 26(1), 21-37.

D'Hondt, E., Deforche, B., Gentier, I., De Bourdeaudhuij, I., Vaeyens, R., Philippaerts, R., \& Lenoir, M. (2013). A longitudinal analysis of gross motor coordination in overweight and obese children versus normal-weight peers. International journal of obesity, 37(1), 61 .

D'Hondt, E., Deforche, B., Gentier, I., Verstuyf, J., Vaeyens, R., De Bourdeaudhuij, I., ... \& Lenoir, M. (2014). A longitudinal study of gross motor coordination and weight status in children. Obesity, 22(6), 1505-1511.

D'Hondt, V., Deforche, B., Vaeyens, R., Vandorpe, B., Vandendriessche, J., Pion, J., ... \& Lenoir, M. (2011). Gross motor coordination in relation to weight status and age in 5-to 12-year-old boys and girls: a cross-sectional study. International journal of pediatric obesity, 6(sup3), e556-564.

Gaya, A. (2008). Ciências do movimento humano: introdução à metodologia da pesquisa.Porto Alegre: Artmed, 2008

Gazolla, F., Bordallo, M. A., Madeira, I., Carvalho, C., Collett-Solberg, P., Bordallo, A. P., ... \& da Cunha, C. (2014). Fatores de risco cardiovasculares em crianças obesas. Revista Hospital Universitário Pedro Ernesto, 13(1).

Gentier, I., D’Hondt, E., Shultz, S., Deforche, B., Augustijn, M., Hoorne, S., ... \& Lenoir, M. (2013). Fine and gross motor skills differ between healthy-weight and obese children. Research in Developmental Disabilities, 34(11), 4043-4051.

Hannon, T. S., Rao, G., \& Arslanian, S. A. (2005). Childhood obesity and type 2 diabetes mellitus. Pediatrics, $116(2)$, 473-480.

Kussuki, M. O. M., João, S. M. A., \& da Cunha, A. C. P. (2007). Caracterização postural da coluna de crianças obesas de 7 a 10 anos. Fisioterapia em movimento, 20(1). 
Logan, S. W., Scrabis-Fletcher, K., Modlesky, C., \& Getchell, N. (2011). The relationship between motor skill proficiency and body mass index in preschool children. Research quarterly for exercise and sport, 82(3), 442-448.

Lopes, V. P., Stodden, D. F., Bianchi, M. M., Maia, J. A., \& Rodrigues, L. P. (2012). Correlation between BMI and motor coordination in children. Journal of Science and Medicine in Sport, 15(1), 38-43.

Marmeleira, J., Veiga, G., Cansado, H., \& Raimundo, A. (2017). Relationship between motor proficiency and body composition in 6 - to 10 - year - old children. Journal of paediatrics and child health, 53(4), 348-353.

Miranda, T. B., Beltrame, T. S., \& Cardoso, F. L. (2011). Desempenho motor e estado nutricional de escolares com e sem transtorno do desenvolvimento da coordenação. Revista Brasileira de Cineantropometria e Desempenho Humano, 13(1), 59-66.

Nunez-Gaunaurd, A., Moore, J. G., Roach, K. E., Miller, T. L., \& Kirk-Sanchez, N. J. (2013). Motor proficiency, strength, endurance, and physical activity among middle school children who are healthy, overweight, and obese. Pediatric Physical Therapy, 25(2), 130-138.

Okely, A. D., \& Booth, M. L. (2004). Mastery of fundamental movement skills among children in New South Wales: prevalence and sociodemographic distribution. Journal of science and medicine in sport, 7(3), 358-372.

Okely, A. D., Booth, M. L., \& Chey, T. (2004). Relationships between body composition and fundamental movement skills among children and adolescents. Research quarterly for exercise and sport, 75(3), 238-247.

Pereira, É. F., Graup, S., Lopes, A. D. S., Borgatto, A. F., \& Daronco, L. S. E. (2009). Percepção da imagem corporal de crianças e adolescentes com diferentes níveis socio-econômicos na cidade de Florianópolis, Santa Catarina, Brasil. Rev. bras. saúde matern. infant, 9(3), 253-262.

Petrolini, N., Iughetti, L., \& Bernasconi, S. (1995). Difficulty in visual motor coordination as a possible cause of sedentary behaviour in obese children. International journal of obesity and related metabolic disorders: journal of the International Association for the Study of Obesity, 19(12), 928-928.

Poulsen, A. A., Desha, L., Ziviani, J., Griffiths, L., Heaslop, A., Khan, A., \& Leong, G. M. (2011). Fundamental movement skills and self-concept of children who are overweight. International Journal of Pediatric Obesity, 6(sup3), e464-471.

Rosaneli, C. F., Baena, C. P., Auler, F., Nakashima, A. T. A., Netto-Oliveira, E. R., Oliveira, A. B., ... \& Faria-Neto, J. R. (2014). Aumento da pressão arterial e obesidade na infância: uma avaliação transversal de 4.609 escolares. Arq Bras Cardiol, 103(3), 238-44.

Sibella, F., Galli, M., Romei, M., Montesano, A., \& Crivellini, M. (2003). Biomechanical analysis of sit-to-stand movement in normal and obese subjects. Clinical biomechanics, 18(8), 745-750.

Simmonds, M., Llewellyn, A., Owen, C. G., \& Woolacott, N. (2016). Predicting adult obesity from childhood obesity: a systematic review and meta - analysis. Obesity reviews, 17(2), 95-107.

Swinburn, B. A., Sacks, G., Hall, K. D., McPherson, K., Finegood, D. T., Moodie, M. L., \& Gortmaker, S. L. (2011). The global obesity pandemic: shaped by global drivers and local environments. The Lancet, 378(9793), 804-814.

Wade, M. G., \& Whiting, H. T. A. (Eds.). (1986). Motor development in children: Aspects of coordination and control. Dordrecht: Martinus Nijhoff Publishers, p. 341-60.

World Health Organization. (2004). Diabetes action now: an initiative of the World Health Organization and the International Diabetes Federation. https://apps.who.int/iris/handle/10665/42934.

Zanella, L. W., Bandeira, P. F. R., de Souza, M. S., \& Valentini, N. C. (2015). Há associação entre o desempenho motor e estado nutricional de escolares?. Conexões, 13(2), 136-148.

Zequinão, M., Medeiros, P., Santos, J. O. L., Silva, I. P., Pereira, B. O., \& Cardoso, F. L. (2015). Relação entre desempenho motor e variáveis antropométricas em crianças e adolescentes de Florianópolis, SC/Brasil. Estudos em Desenvolvimento Motor da Criança 8(1), 18-23. 